OPEN ACCESS

Edited by: Jinwei Zhang,

University of Exeter, United Kingdom

Reviewed by:

Carlos Padilha

Federal University of Rio Grande do

Norte, Brazil

Guolin Cai,

Jiangnan University, China

*Correspondence:

Xuehong Chen

chen-xuehong@163.com

Specialty section:

This article was submitted to

Microbiotechnology,

a section of the journal

Frontiers in Microbiology

Received: 21 October 2020

Accepted: 08 February 2021

Published: 24 February 2021

Citation:

Zheng Q, Meng X, Cheng M, Li Y,

Liu Y and Chen X (2021) Cloning and Characterization of a New Chitosanase From a Deep-Sea

Bacterium Serratia sp. QD07.

Front. Microbiol. 12:619731.

doi: 10.3389/fmicb.2021.619731

\section{Cloning and Characterization of a New Chitosanase From a Deep-Sea Bacterium Serratia sp. QD07}

\author{
Qiuling Zheng', Xiangjun Meng' ${ }^{2}$, Mingyang Cheng ${ }^{1}$, Yanfeng Li', Yuanpeng Liu' ${ }^{1}$ and \\ Xuehong Chen ${ }^{1 *}$
}

'Department of Pharmacology, School of Basic Medicine, Qingdao University, Qingdao, China, ${ }^{2}$ Qingdao Mental Health Center, Qingdao, China

Chitosanase is a significant chitosan-degrading enzyme involved in industrial applications, which forms chitooligosaccharides (COS) as reaction products that are known to have various biological activities. In this study, the gene csnS was cloned from a deep-sea bacterium Serratia sp. QD07, as well as over-expressed in Escherichia coli, which is a new chitosanase encoding gene. The recombinant strain was cultured in a $5 \mathrm{~L}$ fermenter, which yielded $324 \mathrm{U} / \mathrm{mL}$ chitosanases. After purification, CsnS is a cold-adapted enzyme with the highest activity at $60^{\circ} \mathrm{C}$, showing $37.5 \%$ of the maximal activity at $0^{\circ} \mathrm{C}$ and $42.6 \%$ of the maximal activity at $10^{\circ} \mathrm{C}$. It exhibited optimum activity at $\mathrm{pH} 5.8$ and was stable at a pH range of 3.4-8.8. Additionally, CsnS exhibited an endotype cleavage pattern and hydrolyzed chitosan polymers to yield disaccharides and trisaccharides as the primary reaction products. These results make CsnS a potential candidate for the industrial manufacture of COS.

Keywords: chitosanase, chitooligosaccharides, deep-sea bacterium, Serratia sp. QD07, fermenter

\section{INTRODUCTION}

Chitosan, the primary deacetylation product of chitin, is a linear cationic polysaccharide comprising of $\beta$-(1,4)-linked D-glucosamine (GlcN or D unit) and $N$-acetyl-D-glucosamine (GlcNAc or A unit) (Muanprasat and Chatsudthipong, 2017). The chitosan polymer is a natural alkaline polysaccharide, insoluble in most neutral liquids such as water (Naveed et al., 2019). Chitooligosaccharide (COS), the degradation product of chitosan, has aroused an increasing interest due to its excellent biological properties and potential applications. Of particular importance are its admirable biological activities including anti-microbial (Fernandes et al., 2008), anti-fungal (Mei et al., 2015), anti-oxidant (Ma et al., 2020), anti-inflammatory (Kunanusornchai et al., 2016), anti-obesity (Huang et al., 2015), anti-tumor (Suzuki et al., 1986; Zhao et al., 2019), anti-hypertensive (Huang et al., 2005), anti-HIV-1 (Artan et al., 2010), anti-Alzheimer's (Eom et al., 2013), and immune-enhancing effects (Zhang et al., 2014). It also shows promise as a drug/DNA delivery agent (Kumari et al., 2018; Li et al., 2019a; Wang et al., 2020). Therefore, COS is a promising candidate with potential application in several fields, including the medical and pharmaceutical industries.

Chitosanases (EC 3.2.1.132) are divided into five families: GH-5, GH-8, GH-46, GH-75, and GH-80 in the CAZy database (Thadathil and Velappan, 2014). Families GH-5 and GH-8 could hydrolyze chitosan and some other glycosides, while the families GH-46, GH-75 and GH-80 
comprise chitosanases merely (Johnsen et al., 2010). Chitosanases catalyzed the breaking of the $\beta-(1,4)$ glycosidic bonds in chitosan to yield low molecular weight chitosan (LMWC) or COS with different Degree of Polymerization (DP) (Brzezinski, 2011). Due to the substrate specificity of different chitosanases, the degradation of COS by different DP resulted in different biological activities. For example, the DP 1-3 inhibits matrix metalloproteinase-9 in human fibrosarcoma cells (Van Ta et al., 2006); the DP 2-6 (Qiao et al., 2011) exhibit anti-inflammatory activity; the DP6 shows anti-tumor activity (Xiong et al., 2009), whereas the DP 8-12 have higher anti-oxidant activity (Li et al., 2012).

Currently, COS can be produced by chemical, physical, electrochemical and enzymatic methods (Liang et al., 2018). Owing to the lack of contaminants and the easily controllable nature of the process, using chitosanases are relatively satisfactory tools to produce COS in enzymatic methods. Considering the lack of a suitable commercial chitosanase for the production of COS, we previously established an efficient affinity purification method to rapidly detect chitosanase in bacterial cultural supernatant (Li et al., 2019b), and several chitosanase-producing marine bacteria strains including a deep-sea bacterium Serratia sp. QD07 were successfully isolated. Chitosanase is mainly derived from bacteria (Luo et al., 2020), but to a lesser extent from fungi (Wang et al., 2008), plant tissues (Osswald et al., 1994) and viruses (Blanc et al., 2014). Although many chitosanases have been reported, there are only a few chitosanases with cold adaptability (Johnsen et al., 2010; Qin et al., 2018; Yang et al., 2019). The yield of chitosanase of microorganisms reported so far is different.

In this study, the gene $\operatorname{cs} n S$ was cloned from Serratia sp. QD07, as well as expressed in Escherichia coli, which is a chitosanase encoding gene. Additionally, an efficient fermentation and purification method was established and the biochemical features of CsnS were characterized.

\section{RESULTS AND DISCUSSION}

\section{Sequence Analysis of CsnS}

The deep-sea bacterium Serratia sp. QD07 was isolated from a sample of sea mud in the South China Sea (depth: $1179 \mathrm{~m}$ ). This strain proliferated rapidly in the selection medium containing chitosan $[0.5 \%(\mathrm{w} / \mathrm{v})]$ and showed high chitosanases activity. Results of the sequence analysis showed that its genome contained a putative chitosanase-encoding gene, csnS. In this study, the csnS gene was cloned from Serratia sp. QD07, which consisted of an intact open reading frame (ORF) of 756 base pairs, and an encoded protein, CsnS containing 251 amino acid residues. Signal peptide analysis revealed that the $N$-terminal of CsnS lacked signal peptide. The theoretical pI of CsnS was 5.56, as well as the molecular weight (MW) of CsnS was $27.1 \mathrm{kDa}$.

The phylogenetic tree was established by sequences analysis of CsnS and some other reported chitosanases from families GH-46, GH-75, and GH-80 (Figure 1). CsnS was inferred to belong to the glycoside hydrolyase-46 (GH-46) family. It was found that CsnS showed higher homology with a GH-46 chitosanase (GenBank: AAA19865.1), obtained from a species of the genus Streptomyces. In addition, the conservative domain database (CDD) search was conducted on NCBI, and CsnS was a recognized chitosanase with a conservative domain, belonging to the family GH-46. To further analyze its structure, multiple sequence alignment (MSA) between CsnS and seven other GH46 chitosanases was established. Results from the MSA (Figure 2) showed that the enzyme contains some conserved regions with this chitosanase of family GH-46. The conserved sites Glu35 and Asp53 play vital roles in the hydrolysis of glycosides (Saito et al., 1999; Lyu et al., 2015). The regions 'AENS' (34-37), 'IGFC' (62-65), 'VMHG' (161-164), and sites Tyr47, Ile51, Asp53, Arg55, Thr58, Asp69, and Tyr125 are related to sugar binding (Lyu et al., 2014).

\section{Fermentation and Purification of CsnS}

The first phase of the process commenced with an original glycerol concentration of $10 \mathrm{~g} / \mathrm{L}$. At the beginning of the second stage, when OD 600 value was 1.0 , IPTG $(0.1 \mathrm{mM})$ was added, and the fermentation temperature was reduced to $20^{\circ} \mathrm{C}$. The culture volume used for expression and purification is $50 \mathrm{~mL}$. During the entire process, the feeding was controlled by maintaining the concentration of dissolved oxygen at $30 \%$ of air saturation. Once the enzyme activity decreased, the fermentation process was stopped (Figure 3). At the end of the process at $60 \mathrm{~h}$, the extracellular CsnS activity was calculated to be $324 \mathrm{U} / \mathrm{mL}$.

The recombinant CsnS was purified 1.7-fold using Ni-NTASepharose column chromatography. Its MW was determined by sodium dodecyl sulfate-polyacrylamide gel electrophoresis (SDS-PAGE) to be about $27 \mathrm{kDa}$ (Figure 4), corresponding to the predicted MW. The specific activity of purified CsnS was determined to be $412.6 \mathrm{U} / \mathrm{mg}$. The activity recovery was $91.9 \%$.

\section{The Different Conditions on the Production of CsnS Effects of Different Cell Density OD 600 on the Induction Cultivation Phase on CsnS}

Cell density OD 600 was a critical parameter, which led to a significant effect on the extracellular activity at induction cultivation phase. As shown in Supplementary Figure 1, the induced cells reached 8 and the extracellular activity was $26 \mathrm{U} / \mathrm{mL}$ at OD 600. Whereas, when the induced cells reached 1 , the extracellular activity was the highest, $81 \mathrm{U} / \mathrm{mL}$, which was 3.1fold higher than that of cell density (OD 600) at 8. Moreover, it was 2.1-fold higher than that at 4 of OD 600, which exhibited an extracellular activity of $38 \mathrm{U} / \mathrm{mL}$. The extracellular activity was closer to cell density OD 600 at 1 and 2, after induction, and the extracellular activity of cell density OD 600 at 2 was $71 \mathrm{U} / \mathrm{mL}$ which was lower than OD 600 at 1 . Consequently, at OD 600 of the induced cells reached 1 of OD 600, E. coli was more beneficial to the secretion expression.

\section{Effects of Temperature on the Secretion of CsnS}

Temperature is a key parameter in the production of recombinant proteins in Escherichia coli. Generally, the recombinant E. coli would grow slowly at low temperature, and low production rate always conduced low protein yields. However, the high 


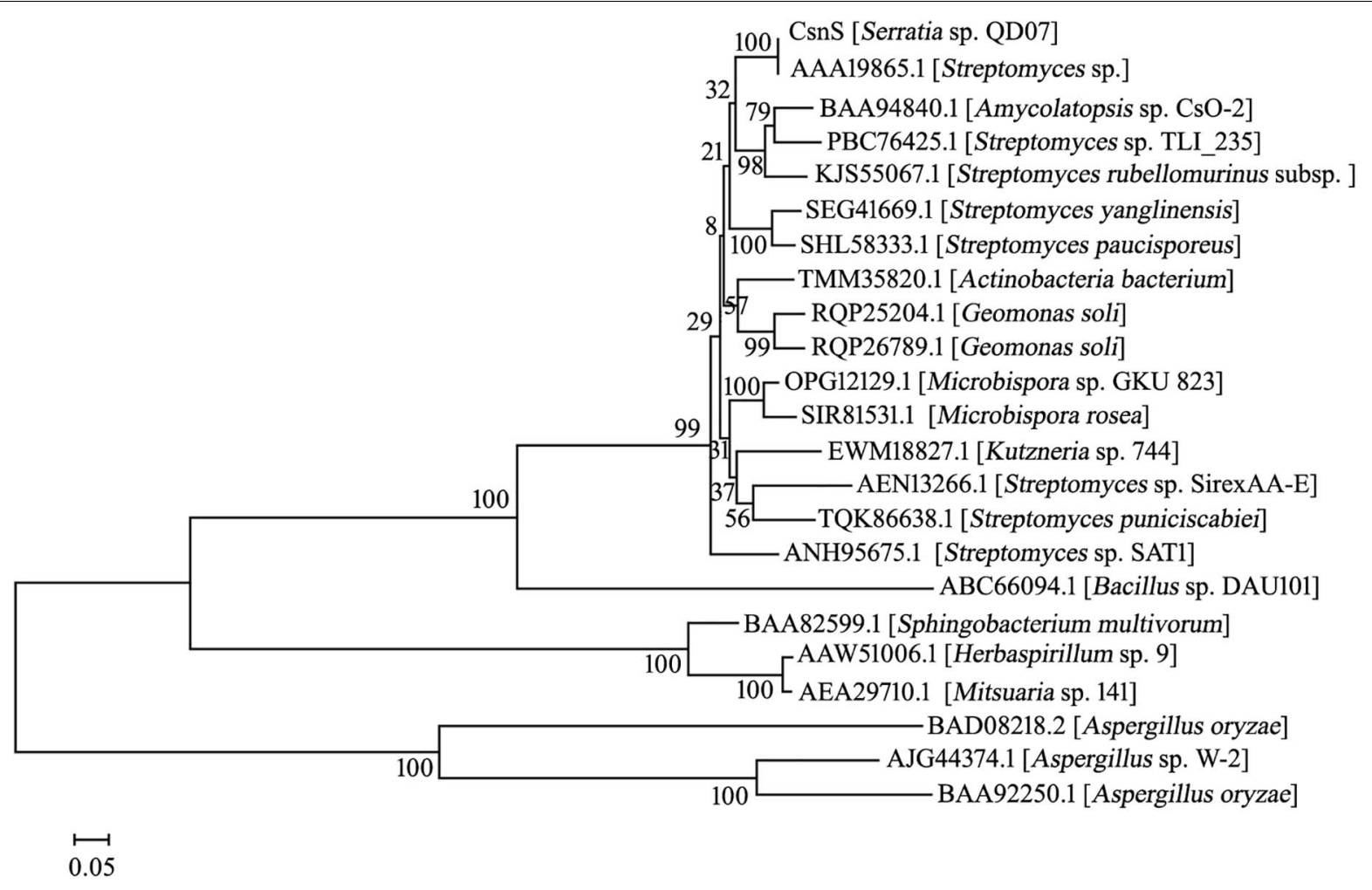

FIGURE 1 | The phylogenetic relationships between CsnS and other chitosanases from GH family 46, 75, and 80 is shown in the neighbor-joining tree. The bootstrap test of the tree repeated 1,000 times.

temperature would denature recombinant proteins and reduce enzyme activities. As shown in Supplementary Figure 2, under the induction temperature of $35^{\circ} \mathrm{C}$, the number of OD 600 induced cells attained 8.9, while the extracellular activity was only $38 \mathrm{U} / \mathrm{mL}$. By contrast, under the induction of $20^{\circ} \mathrm{C}$, the coordination between protein synthesis and translocation was better, and extracellular activity was the highest, $82 \mathrm{U} / \mathrm{mL}$, which was 2.1 -fold higher than that under the induction of $35^{\circ} \mathrm{C}$. The extracellular activity was $58 \mathrm{U} / \mathrm{mL}, 1.4$-fold higher than that of $30^{\circ} \mathrm{C}$. This high yield might be due to the low temperature which reduced the denaturation rate of the target proteins and allowed the freshly synthesized peptides to fold correctly. Although $15^{\circ} \mathrm{C}$ might be more suitable for CsnS production, the significantly lowered cell density limited its total protein yield. Consequently, E. coli was more favorable for secretion expression at a lower temperature of $20^{\circ} \mathrm{C}$.

\section{Effects of Concentration of IPTG on the Secretion of Csns}

The IPTG was added to induce the protein of CsnS expression. Actually, the IPTG was potentially toxic chemical that restrained cell growth with increasing concentration. Experiments were performed to investigate and identify the optimal induction concentration of IPTG. As shown in Supplementary Figure 3, at an induction concentration of $0.8 \mathrm{mM}, \mathrm{OD} 600$ of the induced cells reached only 6.1, but the extracellular activity was also low and only $36 \mathrm{U} / \mathrm{mL}$. When the induced concentration was $0.1 \mathrm{mM}$, the extracellular activity was the highest, $86 \mathrm{U} / \mathrm{mL}, 2.3$-fold higher than that of $0.8 \mathrm{mM}$. Moreover, this activity was 1.4 -fold higher than that of $0.4 \mathrm{mM}$, which exhibited the extracellular activity of $60 \mathrm{U} / \mathrm{mL}$. The extracellular activity was closer at induction concentrations of IPTG at $0.1 \mathrm{mM}$ and $0.2 \mathrm{mM}$, and the extracellular activity of induction concentrations at $0.2 \mathrm{mM}$ was $77 \mathrm{U} / \mathrm{mL}$. This low extracellular activity might be due to the low concentration of IPTG at $0.05 \mathrm{mM}$ and high concentration of IPTG at $0.8 \mathrm{mM}$, which causes an inhibitory effect on the growth of E. coli. Consequently, at induction concentration of $0.1 \mathrm{mM}$, E. coli was more beneficial to the secretion expression.

\section{Biochemical Properties of CsnS}

The biochemical properties of recombinant CsnS were determined by the purified enzyme. As shown in Figure 5A, at $60^{\circ} \mathrm{C}$, CsnS showed the highest activity. At the same time, CsnS exhibits $37.5 \%$ and $42.6 \%$ of its maximal activity at $0^{\circ} \mathrm{C}$ and $10^{\circ} \mathrm{C}$, respectively, which is a cold-adapted enzyme. It can retain approximately $40 \%$ of enzyme activity at temperatures ranging from 0 to $100^{\circ} \mathrm{C}$, which indicates the ruggedness of CsnS in extreme temperature conditions. Compared with temperaturesensitive enzymes, this kind of temperature-resistant enzyme has certain advantages in industrial production. On the one hand, the preparation cost of this kind of enzyme preparation is reduced, and the enzyme preparation has high stability, which can maintain the activity for a long time. On the other hand, the requirements for the reactor cooling system are reduced, 


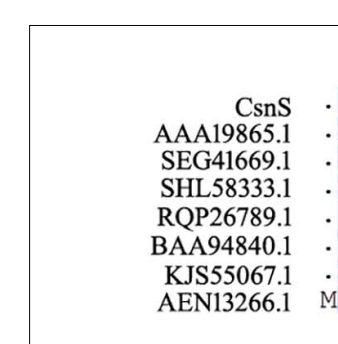

CsnS
AAA19865.1
SEG41669.1
SHL58333.1
RQP26789.1
BAA94840.1
KJS55067.1
AEN13266.1

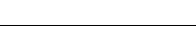

\section{1}

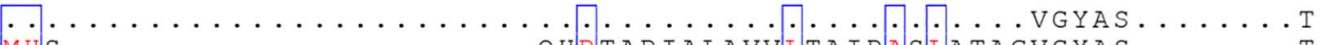
MHS ..................

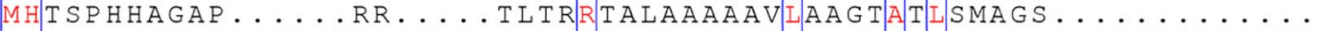
$M H Q S P H G T$ TATT GT T STRRGHRASRPSRRIALIAAAGVIAGGGASIALAGSGQAATPAAAQHRT

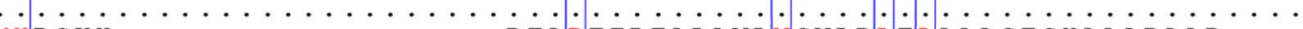
MHPSKH..........................

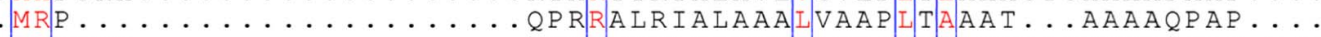

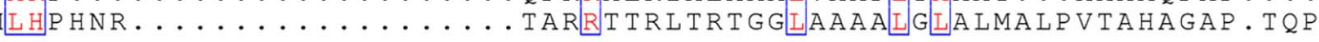

102030

CsnS QA TAVKAGA GLDPH KKEIAMELVSSAENS I I

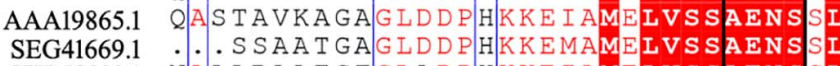

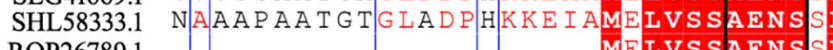
RQP26789.1 : $: \cdots \cdots$

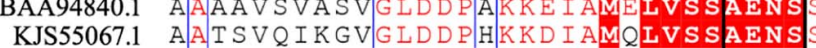

AEN13266.1 A AHHLEAAATGLDDP KKDIAMQLVSSAENS

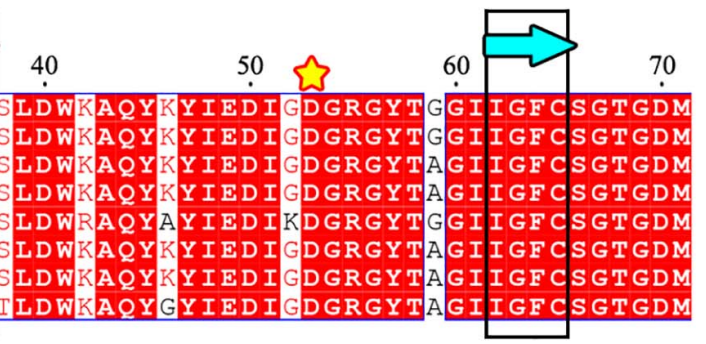

80

90

100

110

120

130

CsnS

AAA19865.1 SEG41669.1 SHL58333.1 RQP26789.1

BAA94840.1

KJS55067.1

AEN13266.1

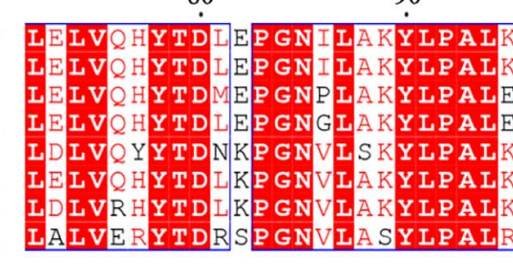

GS $\mathrm{A}$
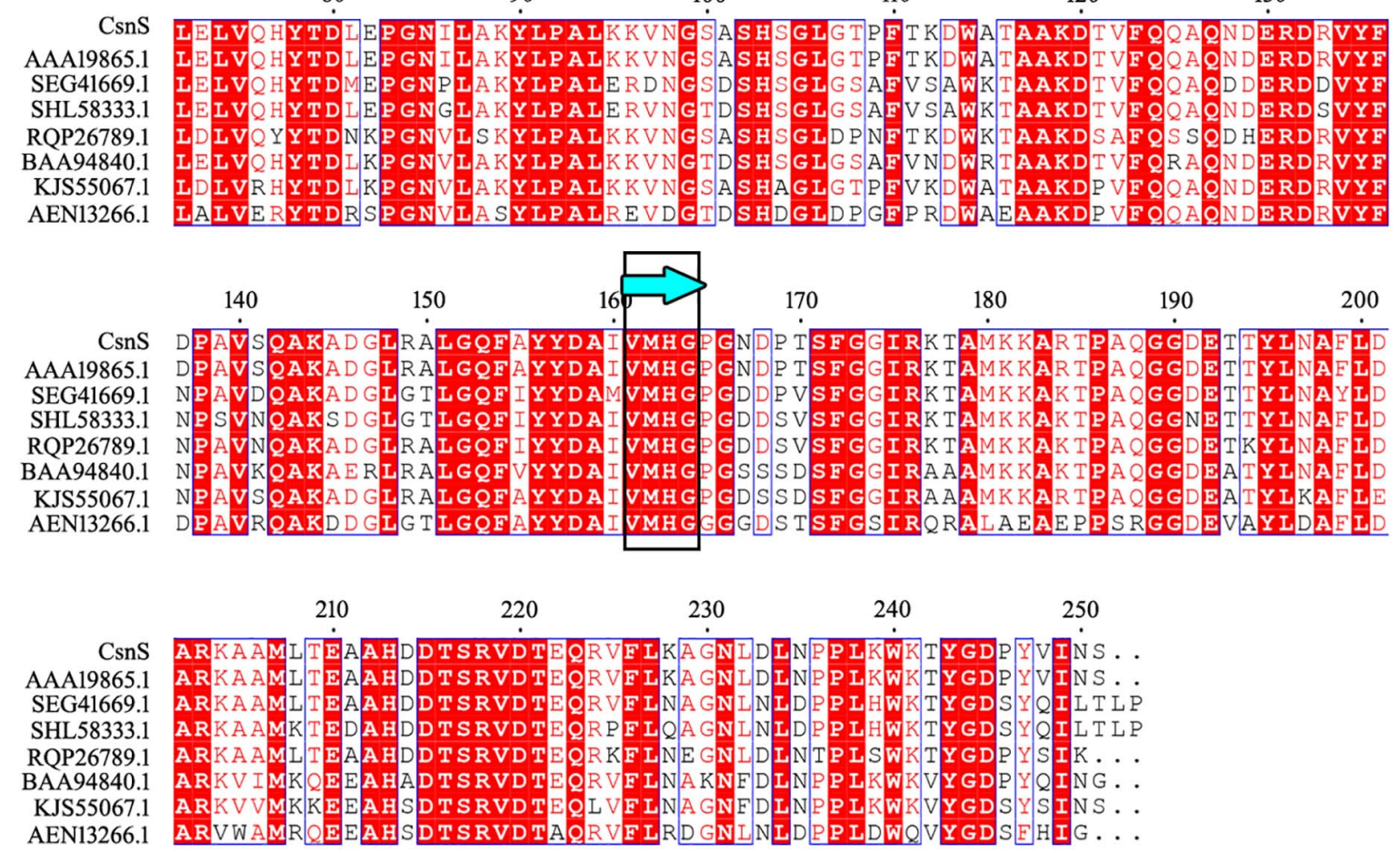

FIGURE 2 | Comparison of the sequence of CsnS with reported chitosanase activity from the GH-46 family: chitosanase from Streptomyces sp. N174 (GenBank number: AAA19865.1); chitosanase from Streptomyces yanglinensis (GenBank number: SEG41669.1); chitosanase from Streptomyces paucisporeus (GenBank number: SHL58333.1); chitosanase from Geomonas soli (GenBank number: RQP26789.1); chitosanase from Amycolatopsis sp. CsO-2 (GenBank number: BAA94840.1); chitosanase from Streptomyces rubellomurinus subsp. indigoferus (GenBank number: KJS55067.1); chitosanase from Streptomyces sp. SirexAA-E (GenBank number: AEN13266.1). The same sugar binding and catalytic sites are marked with blue bands (framed in black) and yellow stars, respectively.

which reduces energy consumption and pollution. In addition, $\mathrm{CsnS}$ has good enzyme activity at $60^{\circ} \mathrm{C}$, and there are few hybrid bacteria living at $60^{\circ} \mathrm{C}$, thus reducing the contamination of bacterial metabolites to products and improving the purity of products. After incubation at temperatures between 0 and $30^{\circ} \mathrm{C}$ for $2 \mathrm{~h}$, the enzyme retained more than $80 \%$ of the initial activity (Figure 5B). These results show that the enzyme can be stored at room temperature and is capable of catalyzing hydrolysis at this temperature. This is a desirable property in industrial applications.

Assays to determine the optimal $\mathrm{pH}$ for CsnS activity were carried out with $0.3 \%(\mathrm{w} / \mathrm{v})$ chitosan at $\mathrm{pH} 4.5-5.7$. CsnS retained more than $60 \%$ of its activity in the experimental range and displayed optimal activity at $\mathrm{pH} 5.2$ (Figure 5C). Additionally, the enzyme can retain more than $80 \%$ activity at $\mathrm{pH} 4.5-5.4$. In order to study $\mathrm{pH}$-stability of the enzyme, various buffers 


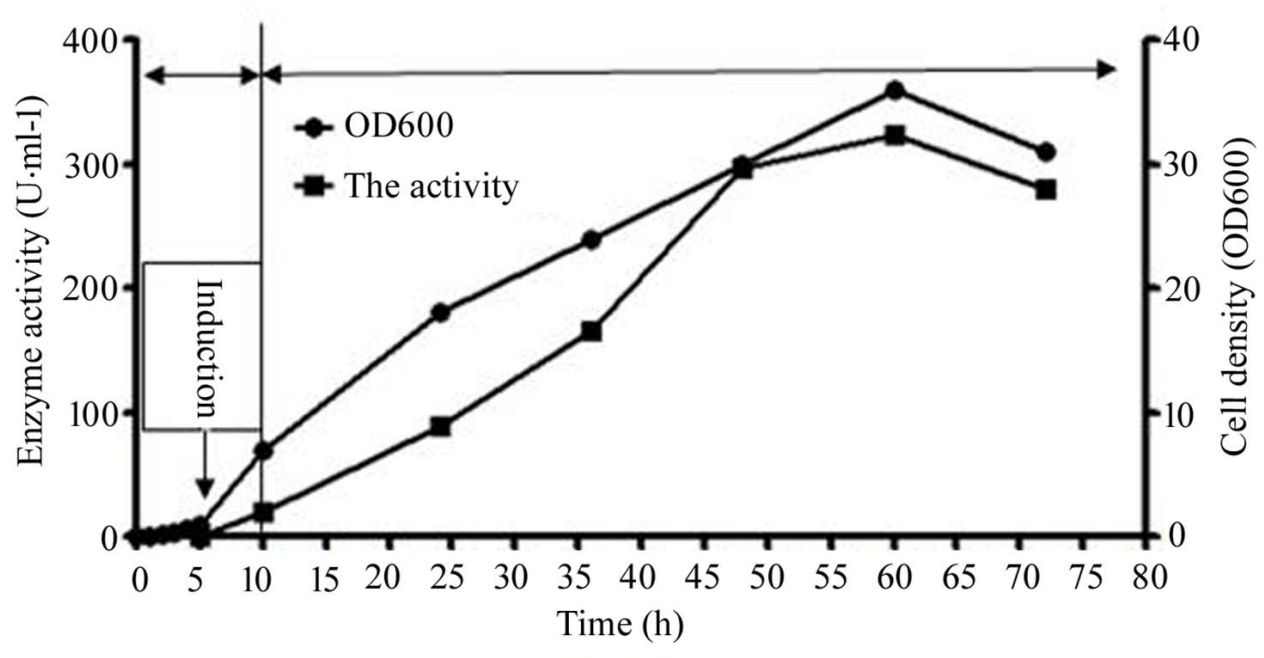

FIGURE 3 | The extracellular activity and cell growth of CsnS production by cultivation. And the entire culture procedure was distributed to three phases: the growth cultivation phase, the induction cultivation phase, and the fed-batch cultivation phase. Since $\mathrm{OD} 600$ reached 1 , the temperature was dropped to $20^{\circ} \mathrm{C}$, as well as IPTG (0.1 mM) was added. Subsequently, the original glycerol was absolutely consumed, the feeding phase of fed-batch cultivation was initiated.

ranging from pH 3.4-9.4 were used in the assays (Figure 5D). The enzyme was found to be stable and retained more than $70 \%$ of its initial activity at a $\mathrm{pH}$ range of 3.4-8.2. At a higher $\mathrm{pH}$ value of 9.4 , it retained about $35 \%$ of its original activity. Therefore, CsnS demonstrates high catalytic properties in either an acidic or alkaline environment and is stable over a broad range of $\mathrm{pH}$. Because the fermentation process of $\mathrm{pH}$ sensing electrode sensor has been delayed, the $\mathrm{pH}$ of the fermentation medium is not fixed during the enzyme production process. Fortunately, $\mathrm{pH}$ has little effect on bacterial growth. Thus, $\mathrm{pH}$ fluctuations do not affect the enzyme activity of chitosanase during the fermentation process.

The effect of metal ions and reagents on the activities of CsnS was shown in Table 1 . We found that except $\mathrm{Cu}^{2+}$ and $\mathrm{Ni}^{2+}$, most metal ions can promote CsnS activity and maintain it above $70 \%$. CsnS shows a strong ability to resist the interference of metal ions. Since most industrial equipments are constructed with metal, the non-reactivity of CsnS to metal ions could facilitate its use in the production process, thereby expanding its application efficiency.

\section{Action Mode and Reaction Product Analysis}

Thin-layer chromatography (TLC) was used to analyze the reaction products and the action modes of CsnS. As shown in Figure 6, no obvious chitooligosaccharides products appeared during the first $30 \mathrm{~min}$ of the reaction. When the substrate was hydrolyzed for $60 \mathrm{~min}$, reaction products were observed, which were mainly a mixture of disaccharides (DP2), trisaccharides (DP3) and tetrasaccharides (DP4). With the extension of reaction time, the relative ratio of DP2 and DP3 oligomers enhanced, whereas those of the higher DP products reduced. Compared with CsnM (Zhou et al., 2019) we studied previously, the product increased DP-1 and DP-4. According to literature reports, DP24 chitosan oligosaccharide has a promising potential for a wide range of applications in the medical field, by virtue of its anti-inflammatory and anti-bacterial activities. These reaction products were similar to most known chitosanases, such as the CsnB from Bacillus sp. BY01 (Yang et al., 2019), CsnQ from Bacillus sp. Q1098 (Ma et al., 2020), Csn-BAC from Bacillus sp. MD-5 (Yang et al., 2020), and Csn-SP from Bacillus sp. DAU101 (Lee et al., 2006 and Table 2), all of which produced $(\mathrm{GlcN})_{2}$ and $(\mathrm{GlcN})_{3}$ as final products. Till date, the main products of chitosanases reported in the literature are mixtures of DP3-DP8, such as chitosanase from Bacillus mycoides TKU038 (Liang et al., 2016) and Bacillus sp. strain KCTC 0377BP (Choi et al., 2004), which are difficult to separate. We foresee CsnS as a potentially valuable agent in the manufacture of easily separable products in industrial production.

\section{MATERIALS AND METHODS}

\section{Materials and Bacterial Cultures}

We purchased E. coli strains BL21 (DE3) as well as expression vector, pET22b (+) from Takara (Dalian, China). From Aladdin Biochemical Technology Co., Ltd. (Shanghai, China), we purchased chitosan (degree of deacetylation $\geq 95 \%$, viscosity: 100-200 mpa.s). And from Merck (Darmstadt, Germany), we purchased the thin-layer chromatography (TLC) silica gel plates.

\section{Isolation of the Bacterial Strains Serratia sp. QD07}

Deep-sea mud samples were collected from South China

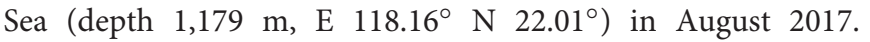
The samples were immersed, diluted, and spread on $2216 \mathrm{E}$ medium. Isolated colonies were cultured on chitosan selective medium containing $0.5 \% \quad(\mathrm{w} / \mathrm{v})$ chitosan, $0.1 \%$ (w/v) KH2PO4, 0.2\% (w/v) K2HPO4, 0.07\% (w/v) MgSO4, 


\section{$\mathrm{kDa} \quad \mathrm{M}$}

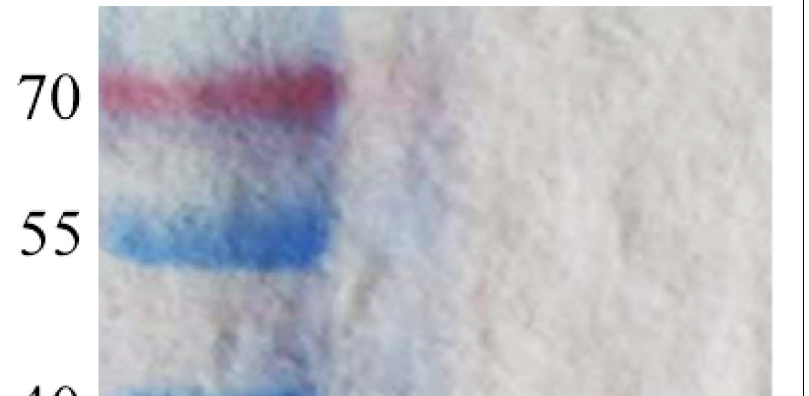

40

35

25

15

\section{0}

FIGURE 4 | SDS-PAGE analysis of the purified CsnS. M, molecular weight markers; 1, purified CsnS.

\section{Sequence Analysis}

The draft genome of Serratia sp. QD07 was determined by using our 2nd generation sequencer. A fictitious chitosanase encoding the gene $\operatorname{csn} S$ was identified and its protein sequence was registered in the GenBank database (Genbank number: MT241387). To further analyze the csnS gene sequence, we used the ORF search program ${ }^{1}$ to determine the open reading frame (ORF). The signal peptide of CsnS via the SignalP 5.0 server $^{2}$ was analyzed. To improve phylogenetic analysis, using the Conserved Domain Database (CDD) to obtain domain and family information. For multi-sequence alignment, we used ClustalX2.1 and ESPript ${ }^{3}$. The phylogenetic tree was constructed by the MEGA 7.0 bootstrap adjacency binding method. In addition, it was using ExPASy ${ }^{4}$ to determine the theoretical isoelectric point (pI) and MW of CsnS.

\section{Expression of Recombinant Chitosanase}

A polymerase chain reaction (PCR) was used to amplify the CsnS fragment without a terminator. The PCR primers used were Ep-CsnS-F and Ep-CsnS-R, which contained the recognition sites $\mathrm{NcoI}$ and XhoI, respectively. The amplified sequence was inserted into plasmid pET22b between the same recognition sites. Escherichiacoli BL21 (DE3) containing the recombinant plasmid pET22b-CsnS plasmid was inoculated into Luria-Bertani (LB) broth for growth at $37^{\circ} \mathrm{C}$ for $6 \mathrm{~h}$.

\section{High-Density Fermentation}

$60 \%$ glycerol stocks of the E. coli BL21-pET22b-CsnS cells were streaked on LB solid medium culture panel supplemented with $50 \mu \mathrm{g} / \mathrm{mL}$ ampicillin and incubated for $26 \mathrm{~h}$ at $37^{\circ} \mathrm{C}$. E. coli colonies containing recombinant plasmid were inoculated in $5 \mathrm{~mL} \mathrm{LB}$ medium containing $50 \mathrm{mg} / \mathrm{mL}$ ampicillin as well as incubated in a rotating shaking table $(180 \mathrm{rpm})$ at $37^{\circ} \mathrm{C}$ overnight. The overnight cultivation $(0.5 \mathrm{~mL})$ was diluted to $50 \mathrm{~mL}$ Terrific Broth Medium (TB) including $50 \mathrm{mg} / \mathrm{mL}$ ampicillin and cultivated on a rotating shaking table $(180 \mathrm{rpm})$ at $37^{\circ} \mathrm{C}$ before the optical density reading at $600 \mathrm{~nm}$ (OD 600) was 8.0. Accordingly, it was cultured in a bioreactor and fermented in a $5 \mathrm{~L}$ fermentation tank (BL Bio-5GJ, Shanghai, China). The $5 \%(\mathrm{v} / \mathrm{v})$ seed culture was inoculated into TB medium. The complete culture process was divided into three stages. At the beginning of the first stage, the initial concentration of glycerol was $10 \mathrm{~g} / \mathrm{L}$ at $37^{\circ} \mathrm{C}$. When the $\mathrm{OD} 600$ reading was 1.0, we moved to the second phase in which, isopropyl $\beta$-d-thiogalactoside (IPTG, $0.1 \mathrm{mM}$ ) was added to induce the protein formation. The cultivation temperature was decreased to $20^{\circ} \mathrm{C}$ and the sample was incubated. When glycerol in the medium was completely consumed, as indicated by a sudden increase in dissolved oxygen (DO) and it was reaching $60 \%$ of air saturation, we commenced the third phase. In this phase, we continued fermentation as a fed-batch with controlled feeding

\footnotetext{
${ }^{1}$ https://www.ncbi.nlm.nih.gov/orffinder/

${ }^{2}$ http://www.cbs.dtu.dk/services/SignalP/

${ }^{3}$ http://espript.ibcp.fr/ESPript/cgi-bin/ESPript.cgi

${ }^{4}$ https://web.expasy.org/cgi-bin/protparam/protparam
} 
A

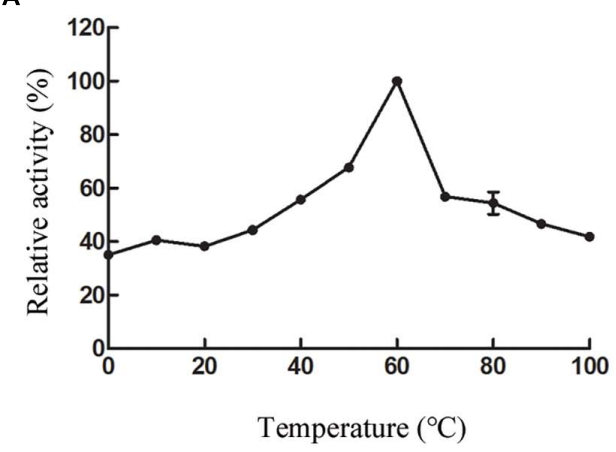

C

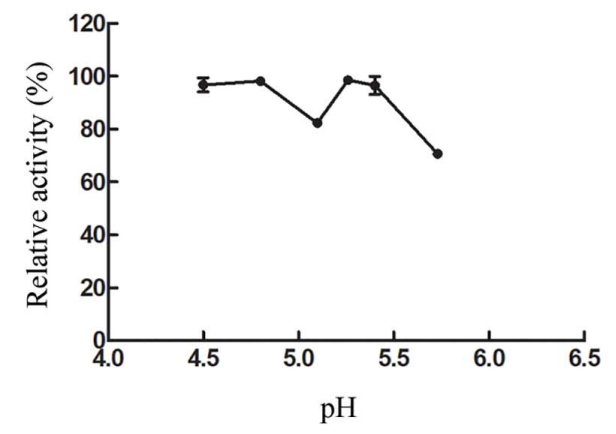

B

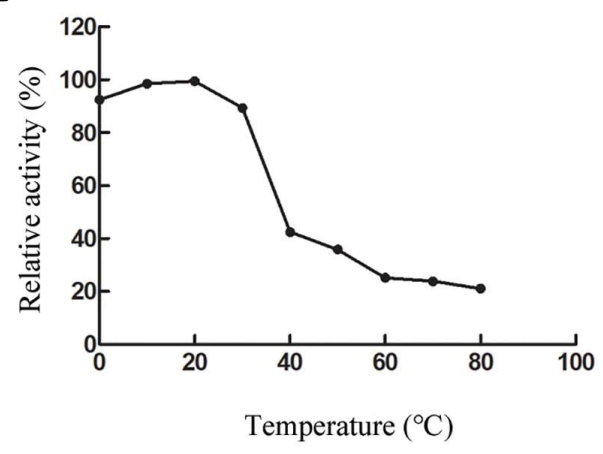

D

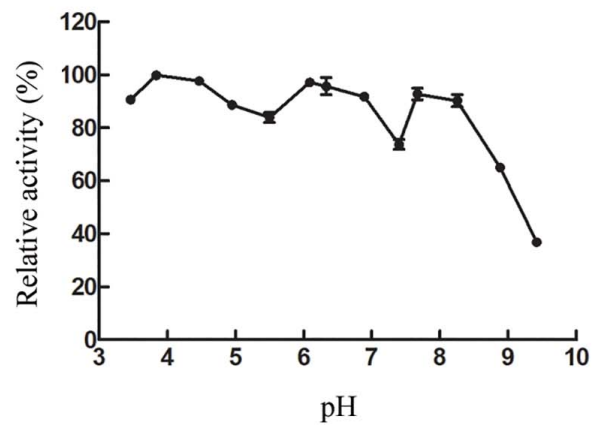

FIGURE 5 | Effect of temperature and pH on CsnS activity. (A) Effect of temperature on the activity of CsnS. (B) Thermal stability of CsnS. Enzyme was incubated for $2 \mathrm{~h}$ at $0-80^{\circ} \mathrm{C}$. (C) Effect of $\mathrm{pH}$ on the activity of CsnS (Sodium acetate buffer $\mathrm{pH} 4.5-5.73$ ). (D) pH stability of CsnS. The $\mathrm{pH}$ stability was analyzed by measuring the residual activity after pretreating the enzyme in different buffers at $4^{\circ} \mathrm{C}$ for $48 \mathrm{~h}$ (citric acid/Na2HPO4 buffer, pH 2.9-6.09; Na2HPO4/NaH2PO4 buffer, pH 6.33-7.40; Tris-HCl buffer, $\mathrm{pH}$ 7.67-8.25; glycine-NaOH buffer, $\mathrm{pH}$ 8.88-9.42). Data represent mean \pm SD from three independent experiments.

to maintain a constant concentration of DO at $30 \%$ of air saturation. In the entire procedure, the $\mathrm{pH}$ was maintained at 7.0 throughout the addition of $20 \%(\mathrm{v} / \mathrm{v})$ ammonia solution. The speed of cascade impeller was kept between 300 and $500 \mathrm{rpm}$, and the air source used compressed air with an airflow of $6 \mathrm{vvm}$.

\section{Purification of Recombinant CsnS}

The crude enzyme in the supernatant was harvested and centrifuged at $13,000 \mathrm{~g}$ at $4^{\circ} \mathrm{C}$ for $15 \mathrm{~min}$. Phosphate buffers A $(\mathrm{pH} 8.0,500 \mathrm{mM} \mathrm{NaCl})$ and $\mathrm{B}(\mathrm{pH} 8.0,500 \mathrm{mM} \mathrm{NaCl}$, $500 \mathrm{mM}$ iminazole) were used in the purification process. Before the crude enzyme was loaded onto an Ni-NTA column in the AKTA150 automatic purification system, five column volumes of buffer A were used to wash the column in order to enable it to load proteins effectively. The sample loading speed was maintained at $1 \mathrm{~mL} / \mathrm{min}$. The target enzyme was eluted with buffer $\mathrm{B}$ by gradient elution. The enzyme purity and molecular mass were determined using SDS-PAGE.

\section{The Fermentation Process of CsnS}

In order to further optimize the fermentation, the recombinant strains containing CsnS were selected. To enhance the extracellular production of recombinant CsnS, we developed the process optimization strategies, as well as the effects of induction culture steps at various cell densities OD 600,

TABLE 1 | Effects of metal ions and reagents on the activity of CsnS.

\begin{tabular}{|c|c|c|}
\hline Reagent added & Concentration (mM) & Relative activity (\%) \\
\hline None & - & $100.0 \pm 0.0$ \\
\hline $\mathrm{MgSO} 4$ & 20 & $138.4 \pm 3.3$ \\
\hline $\mathrm{FeCl}_{3}$ & 20 & $127.0 \pm 3.1$ \\
\hline $\mathrm{BaCl}_{2}$ & 20 & $138.0 \pm 4.4$ \\
\hline $\mathrm{ZnCl}_{2}$ & 20 & $114.3 \pm 2.8$ \\
\hline EDTA & 20 & $131.4 \pm 1.6$ \\
\hline $\mathrm{FeSO}_{4}$ & 20 & $140.3 \pm 2.6$ \\
\hline $\mathrm{KCl}$ & 20 & $135.4 \pm 0.1$ \\
\hline $\mathrm{CuSO}_{4}$ & 20 & $21.5 \pm 0.4$ \\
\hline $\mathrm{Li}_{2} \mathrm{SO}_{4}$ & 20 & $139.7 \pm 3.1$ \\
\hline $\mathrm{CoCl}_{2}$ & 20 & $96.3 \pm 3.8$ \\
\hline SDS & 20 & $119.3 \pm 0.7$ \\
\hline$\left(\mathrm{NH}_{4}\right)_{2} \mathrm{SO}_{4}$ & 20 & $141.4 \pm 4.1$ \\
\hline $\mathrm{Al}_{2}\left(\mathrm{SO}_{4}\right)_{3}$ & 20 & $140.7 \pm 0.8$ \\
\hline $\mathrm{CaCl}_{2}$ & 20 & $139.2 \pm 3.6$ \\
\hline Nicl2 & 20 & $70.5 \pm 0.8$ \\
\hline
\end{tabular}

Activity without addition of chemicals was defined as $100 \%$. Data are shown as means $\pm S D(n=3)$. 


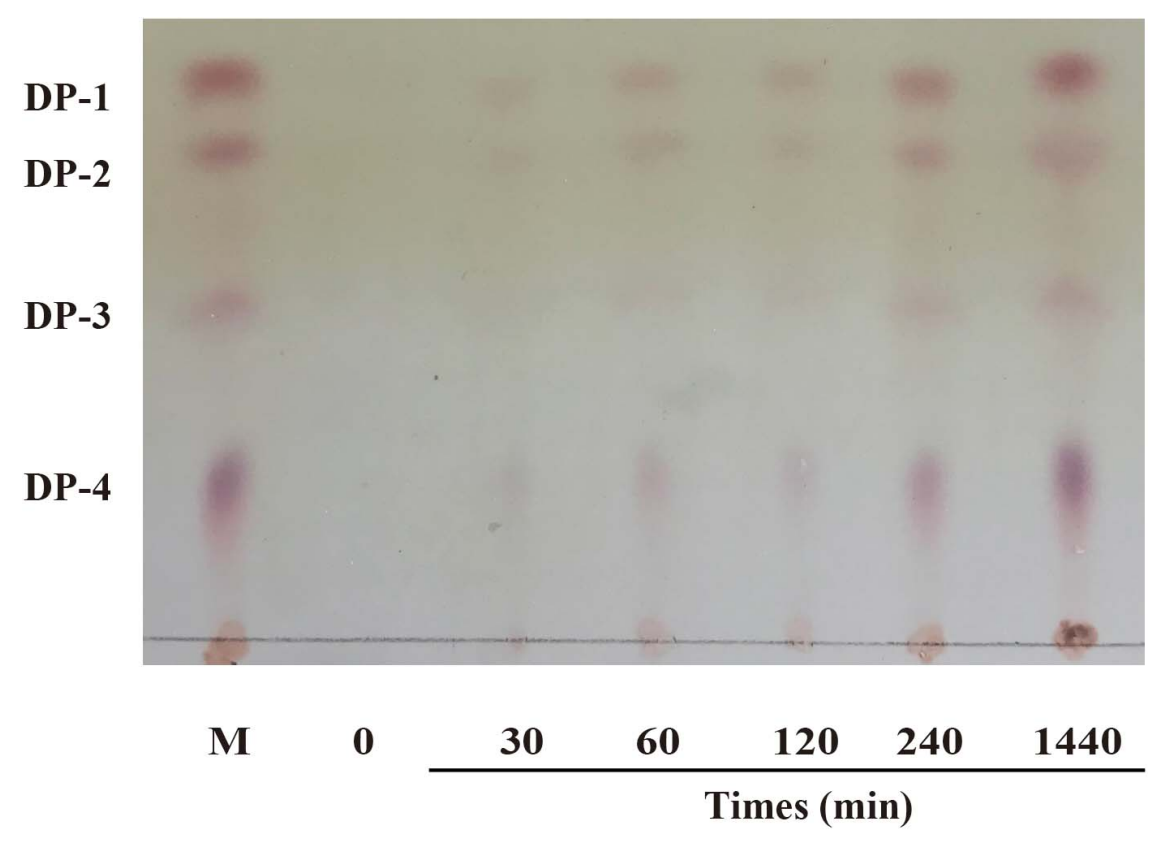

FIGURE 6 | TLC analysis of products after enzymatic hydrolysis.

TABLE 2 | Comparison of the CsnS properties with other reported chitosanases.

\begin{tabular}{|c|c|c|c|c|c|c|c|}
\hline Name & GenBank no. & Orgnism source & Optimal pH & Stable pH range & Optimal temperature $\left({ }^{\circ} \mathrm{C}\right)$ & Products (DP) & References \\
\hline CsnS & MN963773 & Serratia sp. QD101 & 5.8 & $3.4-8.8$ & 60 & $2-3$ & This study \\
\hline CsnQ & MN963773 & Bacillus sp. Q1098 & 5.3 & $6.8-9.1$ & 60 & 2 & Mei et al., 2015 \\
\hline CsnB & MN531545 & Bacillus sp. BY01 & 5.0 & $4.6-5.8$ & 35 & $2-3$ & Liang et al., 2018 \\
\hline Csn-BAC & CP021911 & Bacillus sp. MD-5 & 7.0 & $6.0-8.0$ & 40 & $2-3$ & Li et al., 2019a \\
\hline CS038 & - & Bacillus mycoides TKU038 & 6.0 & $4.0-10.0$ & 50 & $3-9$ & Lyu et al., 2015 \\
\hline CSN-SP & DQ316095 & Bacillus sp. DAU101 & 7.5 & - & 50 & $2-3$ & Saito et al., 1999 \\
\hline chitosanase & AF334682 & Bacillus sp. strain KCTC 0377BP & 5.0 & $4.0-8.0$ & 60 & $3-8$ & Luo et al., 2020 \\
\hline chitosanase & GQ487532 & Janthinobacterium sp. 4239 & 5.0 & - & 45 & $1-2$ & Wang et al., 2020 \\
\hline
\end{tabular}

induction temperatures, and various concentrations of IPTG were investigated.

\section{Effects of Different Cell Density OD 600}

To optimize the cell density OD 600 of induction for CsnS production, Ecoli strain harboring CsnS were induced at 0.5,1, 2,4 , and 8 in TB medium, respectively. The cell was incubated in $50 \mathrm{~mL} \mathrm{~TB}$ including $50 \mathrm{mg} / \mathrm{mL}$ ampicillin and cultured on a rotary shaker $(180 \mathrm{rpm})$ at $37^{\circ} \mathrm{C}$. When the optical density reached at various $600 \mathrm{~nm}$ (OD 600), and then reduced the temperature to $20^{\circ} \mathrm{C}$. Accordingly, IPTG $(0.1 \mathrm{mM})$ was added to induce the expression of target protein, and the cells were incubated for a further $24 \mathrm{~h}$ at $20^{\circ} \mathrm{C}$ sequentially.

\section{Effects of Induction Temperature}

To optimize the induction temperature of CsnS, E.coli strain containing CsnS were induced in TB medium at 15, 20, 25, 30, and $35^{\circ} \mathrm{C}$, respectively. The cell was incubated in $50 \mathrm{~mL}$ TB including $50 \mathrm{mg} / \mathrm{mL}$ ampicillin, as well as cultured on a rotary shaker $(180 \mathrm{rpm})$ at $37^{\circ} \mathrm{C}$ until the optical density at $600 \mathrm{~nm}$
(OD 600) attained 1. Accordingly, we put the shake flasks in a rotary shaker at various induction temperatures. Accordingly, IPTG $(0.1 \mathrm{mM})$ was added to induce the expression of target protein, as well as the cultivation was continued for another $24 \mathrm{~h}$ at $20^{\circ} \mathrm{C}$.

\section{Effects of Different Concentrations of IPTG}

The cells were incubated in $50 \mathrm{~mL} \mathrm{~TB}$ including $50 \mathrm{mg} / \mathrm{mL}$ ampicillin and placed in a rotary shaker $(180 \mathrm{rpm})$ at $37^{\circ} \mathrm{C}$ and $600 \mathrm{~nm}$ (OD 600), until the optical density reached 1, and then dropped the temperature to $20^{\circ} \mathrm{C}$. Various concentrations of IPTG $(0.05,0.1,0.2,0.4$, and $0.8 \mathrm{mM})$ were added to induce the expression of the target protein, and the incubation at $20^{\circ} \mathrm{C}$ for an additional $24 \mathrm{~h}$ sequentially.

\section{Assay of CsnS Activity}

It was using the dinitrosalicylic acid method (DNS) to analyze CsnS activity. After diluting the enzyme in sodium acetate buffer (50 mM, pH 5.8), the enzymatic activity was measured using $100 \mu \mathrm{L}$ enzyme and $900 \mu \mathrm{L} 0.3 \%(\mathrm{w} / \mathrm{v})$ chitosan at $60^{\circ} \mathrm{C}$ for 
$10 \mathrm{~min}$. Next, $0.75 \mathrm{~mL}$ of 3,5-dinitrosalicylic acid was added into the mixture to reduce the reaction. Then, the mixture was boiled for $10 \mathrm{~min}$ and centrifuged to remove the debris, as well as the OD was determined at $520 \mathrm{~nm}$. The mixture with inactivated enzyme constituted the control. One unit (U) of chitosanase was defined as the amount of chitosanase that delivered $1 \mu \mathrm{mol}$ of reducing sugars per min under optimal conditions.

\section{Effect of Temperature, $\mathrm{pH}$ and Metal lons on CsnS}

The enzyme was diluted 200 times with phosphate buffer $(\mathrm{pH}$ 7.0) for biochemical characteristic assays. The substrate chitosan $0.3 \%(\mathrm{w} / \mathrm{v})$ at $\mathrm{pH} 5.8$ was used in the assays to evaluate the influence of temperature and metal ions on the enzyme. The optimal reaction temperature of CsnS was determined, and its activity was measured at various reaction temperatures ranging from 0 to $100^{\circ} \mathrm{C}$. Thermal stability was investigated by measuring the residual activity after pre-incubating the purified enzyme at $0-80^{\circ} \mathrm{C}$ for $2 \mathrm{~h}$. In order to evaluate the effect of metal ions on CsnS, several solutions of metal ions at a final concentration of $20 \mathrm{mM}$ solution were incubated with the purified enzyme at $4^{\circ} \mathrm{C}$ for $48 \mathrm{~h}$ and the enzyme activity was measured. To determine the optimal $\mathrm{pH}$, the substrate chitosan $(0.3 \% \mathrm{w} / \mathrm{v})$ at varying $\mathrm{pH}$ (4.50-5.73) was prepared in sodium acetate buffer. Finally, to determine the effect of $\mathrm{pH}$, the purified enzyme was incubated in various buffers with $\mathrm{pH}$ levels ranging from 3.4 to 9.4.

\section{Analysis of CsnS Degradation Products}

We used TLC to detect degradation products of CsnS, Purified CsnS $(50 \mu \mathrm{L})$ was added to $450 \mu \mathrm{L}$ of chitosan solution $(3 \mathrm{mg} / \mathrm{mL})$ and reacted at $60^{\circ} \mathrm{C}$. The samples were subjected to several reaction times $(10,30,60,120,240$, and 1,440 $\mathrm{min})$. The reaction was terminated by boiling the sample for $10 \mathrm{~min}$. The inactivated samples $(2 \mu \mathrm{L})$ were spotted on to a TLC plate and developed with $n$-propanol/30\% ammonia (2:1) solution. The plates were sprayed with $0.5 \%$ ninhydrin in ethanol, and heated at $100^{\circ} \mathrm{C}$ for $20 \mathrm{~min}$ to visualize sugars.

\section{CONCLUSION}

In this study, CsnS, a new member of GH-46 chitosanase from deep-sea bacterium Serratia sp. QD07 was cloned as well as overexpressed in E. coli. The extracellular production of recombinant CsnS in a $5 \mathrm{~L}$ fermenter indicated a high enzyme activity of $324 \mathrm{U} / \mathrm{mL}$. CsnS is a cold-adapted enzyme, which showed

\section{REFERENCES}

Artan, M., Karadeniz, F., Karagozlu, M. Z., Kim, M. M., and Kim, S. K. (2010). Anti-HIV-1 activity of low molecular weight sulfated chitooligosaccharides. Carbohydr. Res. 345, 656-662. doi: 10.1016/j.carres.2009.12.017

Blanc, G., Mozar, M., Agarkova, I. V., Gurnon, J. R., Yanai-balser, G., Rowe, J. M., et al. (2014). Deep RNA sequencing reveals hidden features and dynamics of early gene transcription in Paramecium bursaria Chlorella virus 1. PLoS One 9:e90989. doi: 10.1371/journal.pone.0090989

Brzezinski, R. (2011). Uncoupling chitosanase production from chitosan. Bioeng. Bugs 2, 226-229. doi: 10.1186/1475-2859-10-7 the highest activity at $60^{\circ} \mathrm{C}$, and exhibited 37.5 and $42.6 \%$ of its maximal activity at $0^{\circ} \mathrm{C}$ and $10^{\circ} \mathrm{C}$, respectively. Hydrolytic product analysis indicated that $\mathrm{CsnS}$ is an endo-type chitosanase, converting chitosan to $(\mathrm{GlcN})_{2}$ and $(\mathrm{GlcN})_{3}$. Therefore, we can conclude that CsnS has suitable properties and may have potential applications in the production of bioactive COS in the food and pharmaceutical industries.

\section{DATA AVAILABILITY STATEMENT}

The original contributions presented in the study are included in the article/Supplementary Material, further inquiries can be directed to the corresponding author/s.

\section{AUTHOR CONTRIBUTIONS}

QZ: formal analysis, writing - original draft preparation, conceptualization, and methodology. XM: editing, analysis, data curation, and software. MC: analysis. YL: software. YL: editing. $\mathrm{XC}$ : funding acquisition and supervision. All authors contributed to the article and approved the submitted version.

\section{FUNDING}

This work was supported by the National Natural Science Foundation of China (No. 81473384) and the Natural Science Foundation of Shandong Province (ZR2018BH036).

\section{SUPPLEMENTARY MATERIAL}

The Supplementary Material for this article can be found online at: https://www.frontiersin.org/articles/10.3389/fmicb. 2021.619731/full\#supplementary-material

Supplementary Figure 1 | Effects of different cell density OD600 in the induction cultivation phase on the extracellular activity in the shake flasks with TB medium at $0.5,1,2,4$, and 8 .

Supplementary Figure 2 | Effects of temperature on the extracellular activity and cell growth Escherichia coli strain which was grown in the shake flasks with TB medium at $15,20,25,30$, and $35^{\circ} \mathrm{C}$.

Supplementary Figure 3 | Effects of different concentrations of IPTG on the extracellular activity and cell growth in Escherichia coli strain which was grown in the shake flasks with TB medium at 0.05, 0.1, 0.2, 0.4, and 0.8 mM. 
Huang, L., Chen, J., Cao, P., Pan, H., Ding, C., Xiao, T., et al. (2015). Anti-obese effect of glucosamine and chitosan oligosaccharide in high-fat diet-induced obese rats. Mar. Drugs 13, 2732-2756. doi: 10.3390/md13052732

Huang, R., Mendis, E., and Kim, S. K. (2005). Improvement of ACE inhibitory activity of chitooligosaccharides (COS) by carboxyl modification. Bioorg. Med. Chem. 13, 3649-3655. doi: 10.1016/j.bmc.2005.03.034

Johnsen, M. G., Hansen, O. C., and Stougaard, P. (2010). Isolation, characterization and heterologous expression of a novel chitosanase from Janthinobacterium sp. strain 4239. Microb. Cell Fact. 9:5. doi: 10.1186/1475-2859-9-5

Kumari, M., Liu, C. H., and Wu, W. C. (2018). Protein moiety in oligochitosan modified vector regulates internalization mechanism and gene delivery: polyplex characterization, intracellular trafficking and transfection. Carbohydr. Polym. 202, 143-156. doi: 10.1016/j.carbpol.2018.08.131

Kunanusornchai, W., Witoonpanich, B., Tawonsawatruk, T., Pichyangkura, R., Chatsudthipong, V., and Muanprasat, C. (2016). Chitosan oligosaccharide suppresses synovial inflammation via AMPK activation: an in vitro and in vivo study. Pharmacol. Res. 113(Pt A), 458-467. doi: 10.1016/j.phrs.2016.09.016

Lee, Y. S., Yoo, J. S., Chung, S. Y., Lee, Y. C., Cho, Y. S., and Choi, Y. L. (2006). Cloning, purification, and characterization of chitosanase from Bacillus sp. DAU101. Appl. Microbiol. Biotechnol. 73, 113-121. doi: 10.1007/s00253-0060444-0

Li, K., Xing, R., Liu, S., Qin, Y., Li, B., Wang, X., et al. (2012). Separation and scavenging superoxide radical activity of chitooligomers with degree of polymerization 6-16. Int. J. Biol. Macromol. 51, 826-830. doi: 10.1016/j. ijbiomac.2012.07.031

Li, S., Wang, L., Chen, X., Sun, M., and Han, Y. (2019a). Design and synthesis of a chitodisaccharide-based affinity resin for chitosanases purification. Mar. Drugs 17:68. doi: 10.3390/md17010068

Li, S., Wang, Y., Li, X., Lee, B. S., Jung, S., and Lee, M. S. (2019b). Enhancing the thermo-stability and anti-biofilm activity of alginate lyase by immobilization on low molecular weight chitosan nanoparticles. Int. J. Mol. Sci. 20:4565. doi: 10.3390/ijms20184565

Liang, S., Sun, Y., and Dai, X. (2018). A review of the preparation, analysis and biological functions of chitooligosaccharide. Int. J. Mol. Sci. 19:2197. doi: 10 . 3390/ijms19082197

Liang, T. W., Chen, W. T., Lin, Z. H., Kuo, Y. H., Nguyen, A. D., Pan, P. S., et al. (2016). An amphiprotic novel chitosanase from Bacillus mycoides and its application in the production of chitooligomers with their antioxidant and antiinflammatory evaluation. Int. J. Mol. Sci. 17:1302. doi: 10.3390/ijms17081302

Luo, S., Qin, Z., Chen, Q., Fan, L., Jiang, L., and Zhao, L. (2020). High level production of a Bacillus amlyoliquefaciens chitosanase in Pichia pastoris suitable for chitooligosaccharides preparation. Int. J. Biol. Macromol. 149, 1034-1041. doi: 10.1016/j.ijbiomac.2020.02.001

Lyu, Q., Shi, Y., Wang, S., Yang, Y., Han, B., Liu, W., et al. (2015). Structural and biochemical insights into the degradation mechanism of chitosan by chitosanase OU01. Biochim. Biophys. Acta 1850, 1953-1961. doi: 10.1016/j. bbagen.2015.06.011

Lyu, Q., Wang, S., Xu, W., Han, B., Liu, W., Jones, D. N., et al. (2014). Structural insights into the substrate-binding mechanism for a novel chitosanase. Biochem. J. 461, 335-345. doi: 10.1042/bj20140159

Ma, C., Li, X., Yang, K., and Li, S. (2020). Characterization of a new chitosanase from a marine Bacillus sp. and the anti-oxidant activity of its hydrolysate. Mar. Drugs 18:126. doi: 10.3390/md18020126

Mei, Y. X., Dai, X. Y., Yang, W., Xu, X. W., and Liang, Y. X. (2015). Antifungal activity of chitooligosaccharides against the dermatophyte Trichophyton rubrum. Int. J. Biol. Macromol. 77, 330-335. doi: 10.1016/j.ijbiomac.2015.03. 042

Muanprasat, C., and Chatsudthipong, V. (2017). Chitosan oligosaccharide: biological activities and potential therapeutic applications. Pharmacol. Ther. 170, 80-97. doi: 10.1016/j.pharmthera.2016.10.013

Naveed, M., Phil, L., Sohail, M., Hasnat, M., Baig, M., Ihsan, A. U., et al. (2019). Chitosan oligosaccharide (COS): an overview. Int. J. Biol. Macromol. 129, 827-843. doi: 10.1016/j.ijbiomac.2019.01.192
Osswald, W. F., Shapiro, J. P., Doostdar, H., Mcdonald, R. E., Niedz, R. P., Nairn, C. J., et al. (1994). Identification and characterization of acidic hydrolases with chitinase and chitosanase activities from sweet orange callus tissue. Plant Cell Physiol. 35, 811-820.

Qiao, Y., Bai, X. F., and Du, Y. G. (2011). Chitosan oligosaccharides protect mice from LPS challenge by attenuation of inflammation and oxidative stress. Int. Immunopharmacol. 11, 121-127. doi: 10.1016/j.intimp.2010.10.016

Qin, Z., Chen, Q., Lin, S., Luo, S., Qiu, Y., and Zhao, L. (2018). Expression and characterization of a novel cold-adapted chitosanase suitable for chitooligosaccharides controllable preparation. Food Chem. 253, 139-147. doi: 10.1016/j.foodchem.2018.01.137

Saito, J., Kita, A., Higuchi, Y., Nagata, Y., Ando, A., and Miki, K. (1999). Crystal structure of chitosanase from Bacillus circulans $\mathrm{MH}-\mathrm{K} 1$ at 1.6-A resolution and its substrate recognition mechanism. J. Biol. Chem. 274, 30818-30825. doi: 10.1074/jbc.274.43.30818

Suzuki, K., Mikami, T., Okawa, Y., Tokoro, A., Suzuki, S., and Suzuki, M. (1986). Antitumor effect of hexa-N-acetylchitohexaose and chitohexaose. Carbohydr. Res. 151, 403-408. doi: 10.1016/s0008-6215(00)90359-8

Thadathil, N., and Velappan, S. P. (2014). Recent developments in chitosanase research and its biotechnological applications: a review. Food Chem. 150, 392-399. doi: 10.1016/j.foodchem.2013.10.083

Van Ta, Q., Kim, M. M., and Kim, S. K. (2006). Inhibitory effect of chitooligosaccharides on matrix metalloproteinase-9 in human fibrosarcoma cells (HT1080). Mar. Biotechnol. 8, 593-599. doi: 10.1007/s10126-006-6031-7

Wang, J., Zhou, W., Yuan, H., and Wang, Y. (2008). Characterization of a novel fungal chitosanase Csn2 from Gongronella sp. JG. Carbohydr. Res. 343, 2583-2588. doi: 10.1016/j.carres.2008.08.004

Wang, Y., Li, S., Jin, M., Han, Q., Liu, S., Chen, X., et al. (2020). Enhancing the thermo-stability and anti-bacterium activity of lysozyme by immobilization on chitosan nanoparticles. Int. J. Mol. Sci. 21:1635. doi: 10.3390/ijms21051635

Xiong, C., Wu, H., Wei, P., Pan, M., Tuo, Y., Kusakabe, I., et al. (2009). Potent angiogenic inhibition effects of deacetylated chitohexaose separated from chitooligosaccharides and its mechanism of action in vitro. Carbohydr. Res. 344, 1975-1983. doi: 10.1016/j.carres.2009.06.036

Yang, G., Sun, H., Cao, R., Liu, Q., and Mao, X. (2020). Characterization of a novel glycoside hydrolase family 46 chitosanase, Csn-BAC, from Bacillus sp. MD-5. Int. J. Biol. Macromol. 146, 518-523. doi: 10.1016/j.ijbiomac.2020.01.031

Yang, Y., Zheng, Z., Xiao, Y., Zhang, J., Zhou, Y., Li, X., et al. (2019). Cloning and characterization of a cold-adapted chitosanase from marine bacterium Bacillus sp. BY01. Molecules 24:3915. doi: 10.3390/molecules24213915

Zhang, P., Liu, W., Peng, Y., Han, B., and Yang, Y. (2014). Toll like receptor 4 (TLR4) mediates the stimulating activities of chitosan oligosaccharide on macrophages. Int. Immunopharmacol. 23, 254-261. doi: 10.1016/j.intimp.2014. 09.007

Zhao, M., Gu, L., Li, Y., Chen, S., You, J., Fan, L., et al. (2019). Chitooligosaccharides display anti-tumor effects against human cervical cancer cells via the apoptotic and autophagic pathways. Carbohydr. Polym. 224:115171. doi: 10.1016/j.carbpol.2019.115171

Zhou, Y., Chen, X., Li, X., Han, Y., Wang, Y., Yao, R., et al. (2019). Purification and characterization of a new cold-adapted and thermo-tolerant chitosanase from marine bacterium Pseudoalteromonas sp. SY39. Molecules 24:183. doi: $10.3390 /$ molecules 24010183

Conflict of Interest: The authors declare that the research was conducted in the absence of any commercial or financial relationships that could be construed as a potential conflict of interest.

Copyright (C) 2021 Zheng, Meng, Cheng, Li, Liu and Chen. This is an open-access article distributed under the terms of the Creative Commons Attribution License (CC BY). The use, distribution or reproduction in other forums is permitted, provided the original author(s) and the copyright owner(s) are credited and that the original publication in this journal is cited, in accordance with accepted academic practice. No use, distribution or reproduction is permitted which does not comply with these terms. 